\title{
Long-read sequencing reveals the complex splicing profile of the psychiatric risk gene CACNA1C in human brain
}

\author{
Michael B. Clark ${ }^{1,2} \cdot$ Tomasz Wrzesinski $^{3}$ - Aintzane B. Garcia ${ }^{1} \cdot$ Nicola A. L. Hall $\mathbb{D}^{1}$ - Joel E. Kleinman ${ }^{4}$. \\ Thomas $\mathrm{Hyde}^{4}$ - Daniel R. Weinberger ${ }^{4}$ - Paul J. Harrison $\mathbb{D}^{1,5} \cdot$ Wilfried Haerty ${ }^{3}$ Elizabeth M. Tunbridge $\mathrm{e}^{1,5}$
}

Received: 12 August 2019 / Revised: 2 October 2019 / Accepted: 25 October 2019 / Published online: 6 November 2019

(c) The Author(s) 2019. This article is published with open access

\begin{abstract}
RNA splicing is a key mechanism linking genetic variation with psychiatric disorders. Splicing profiles are particularly diverse in brain and difficult to accurately identify and quantify. We developed a new approach to address this challenge, combining long-range PCR and nanopore sequencing with a novel bioinformatics pipeline. We identify the full-length coding transcripts of $C A C N A 1 C$ in human brain. CACNAIC is a psychiatric risk gene that encodes the voltage-gated calcium channel $\mathrm{Ca}_{\mathrm{V}}$ 1.2. We show that CACNA1C's transcript profile is substantially more complex than appreciated, identifying 38 novel exons and 241 novel transcripts. Importantly, many of the novel variants are abundant, and predicted to encode channels with altered function. The splicing profile varies between brain regions, especially in cerebellum. We demonstrate that human transcript diversity (and thereby protein isoform diversity) remains undercharacterised, and provide a feasible and cost-effective methodology to address this. A detailed understanding of isoform diversity will be essential for the translation of psychiatric genomic findings into pathophysiological insights and novel psychopharmacological targets.
\end{abstract}

These authors contributed equally: Michael B. Clark, Tomasz Wrzesinski

These authors contributed equally: Wilfried Haerty, Elizabeth M. Tunbridge

Supplementary information The online version of this article (https:// doi.org/10.1038/s41380-019-0583-1) contains supplementary material, which is available to authorized users.

Wilfried Haerty

Wilfried.Haerty@earlham.ac.uk

$\triangle$ Elizabeth M. Tunbridge

elizabeth.tunbridge@psych.ox.ac.uk

1 Department of Psychiatry, University of Oxford, Oxford, UK

2 Centre for Stem Cell Systems, Department of Anatomy and Neuroscience, The University of Melbourne, Melbourne, VIC, Australia

3 The Earlham Institute, Norwich, UK

4 The Lieber Institute for Brain Development, Baltimore, MD, USA

5 Oxford Health NHS Foundation Trust, Oxford, UK

\section{Introduction}

Genomic studies have identified numerous common single nucleotide polymorphisms (SNPs) that are robustly associated with psychiatric disorders; the challenge is now to understand the underlying pathophysiological mechanisms [1]. Most of these SNPs are non-coding [2-4], implying that they mediate disease associations by influencing aspects of RNA expression. Of the various mechanisms of RNA regulation, the possibility that psychiatric risk SNPs might influence RNA splicing is particularly appealing, given its exquisite, cell type-specific regulation and its key role in determining neuronal properties [5]. Consistent with this hypothesis, at a global level, cellular studies emphasise RNA splicing as a key mechanism mediating the effect of disease-associated, non-coding variants in complex disorders, including schizophrenia [6]. Similarly, in human brain, genomic regions associated with schizophrenia are enriched for genes that show differential isoform usage across neurodevelopment [7], implicating many schizophrenia-associated risk loci in the regulation of the expression of specific RNA transcripts. Consistent with these findings, examples of associations between psychiatric risk-associated loci and the abundance of novel, 
alternatively spliced transcripts are beginning to emerge [8-10], complementing numerous reports of altered splicing patterns in the brains of psychiatric cases, compared with controls [11-13].

Splicing varies extensively across human development and aging [7], and between tissues. The brain exhibits one of the highest levels of splicing diversity and prominent use of tissue-specific exons, microexons and splicing factors [14-18]. However, despite extensive efforts to improve the annotation of the human genome [19], the alternative splicing patterns of many genes remain poorly described, especially in brain, and novel coding exons (many of which are enriched within brain-expressed genes [20, 21]) and transcripts are continually being discovered. This is due to the limited availability of high-quality, post-mortem human tissue and to technical limitations associated with standard approaches: most rely on short-read RNA-Seq and the reconstruction of fragmented sequences making the disambiguation of full-length transcripts difficult, particularly for large and complex genes. Benchmarking studies have shown that short-read RNA-Seq methodologies are unable to accurately reconstruct and quantitate the majority of transcript and protein isoforms [22, 23].

The relative lack of knowledge about the splicing of individual human genes is typified by CACNA1C. CAC$N A I C$ encodes the $\mathrm{Ca}_{\mathrm{v}} 1.2$ voltage-gated calcium channel (VGCC) alpha ${ }_{1}$ subunit and is a leading genomically informed candidate gene for multiple psychiatric disorders $[2,3,24,25]$. However, it is not known how its expression and/or splicing is altered in illness, or in association with genetic risk for it [26]. The CACNAIC gene is large (cDNA 13433 nucleotides [nt], 2209 amino acids [aa]) and complex, with at least 50 annotated exons and 31 predicted transcripts (Gencode 27; Fig. 1a) [17]. Its size and complexity make accurate transcript identification and quantification by standard gene expression measures extremely difficult; consequently, the true full-length protein sequence of isoforms remains unclear. Information on the transcript diversity of human neuronal VGCC subunits is sparse: most studies have focused on rodents [18], or human cardiac tissue [27, 28]; the sole study examining VGCC splicing in human brain used a single adult sample [29]. This information is significant, both in terms of understanding pathophysiological mechanisms and for the development of novel pharmacological agents, because CACNAIC encodes multiple alternatively spliced transcripts, which result in functionally and pharmacologically distinct channels [30, 31].

To address these unknowns, we developed a straightforward and cost-effective approach using a long-read sequencing technology [32-34]. We combined long-range RT-PCR with long-read nanopore sequencing and a novel bioinformatics pipeline to characterise full-length
CACNAIC coding sequences (CDSs) in post-mortem human brain. We show that the transcript structure of human CACNAIC is substantially more complex than currently appreciated, with numerous novel transcripts and isoforms containing unannotated exons, novel splicing junctions, and in-frame deletions that are predicted to alter the protein sequence and function.

\section{Methods}

\section{Sample preparation and sequencing}

Post-mortem brain tissue from three adult donors in the Lieber Institute for Brain Development repository was obtained from the Maryland Medical Examiner's Office [35]. Subjects with evidence of neuropathology, drug use (other than alcohol), or psychiatric illness were excluded. Demographic information is presented in Supplementary Table 1.

Full methodological details are presented in the Supplementary Information. Briefly, RNA was extracted from cerebellum, striatum, dorsolateral prefrontal cortex [DLPFC], cingulate cortex, occipital cortex and parietal cortex and reverse transcribed. We selected these brain regions to investigate transcript diversity in cortical and subcortical regions known to be transcriptionally and functionally diverse [36] and to investigate to what extent the CACNAIC transcript profile differs between cortical regions. Full-length CDSs (from the first to last exon [focussing on 'Exon1B' the start exon most abundant in brain [30]]) were amplified using PCR, and barcoded and sequenced (across two runs; six samples common to both runs) using Oxford Nanopore Technology's MinION.

\section{Data analysis and code availability}

Our novel data analysis pipeline is available from: https:// github.com/twrzes/TAQLoRe. 2D pass reads (i.e. those with a $q$-score of $\geq 9$ ) with an identified barcode were mapped to the transcriptome (hg38, ENSEMBL v82) using a HPC version of BLAT (pblat-cluster v0.3, available from http://icebert.github.io/pblat-cluster/ [37]) and to the $C A C$ $N A I C$ metagene containing known exonic sequences using GMAP version 2017-04-24 [38]. Reads mapping uniquely to $C A C N A 1 C$, and with at least $50 \%$ of their length mapped were retained for further analysis.

To identify potential novel exons, we mined the alignments of the reads to the transcriptome for inserts of at least nine nucleotides in length located at the junctions between annotated exons. To further characterize candidate novel exons, we mapped the corresponding reads to the genome using LAST v926 [39]. We retained candidate exons 
A

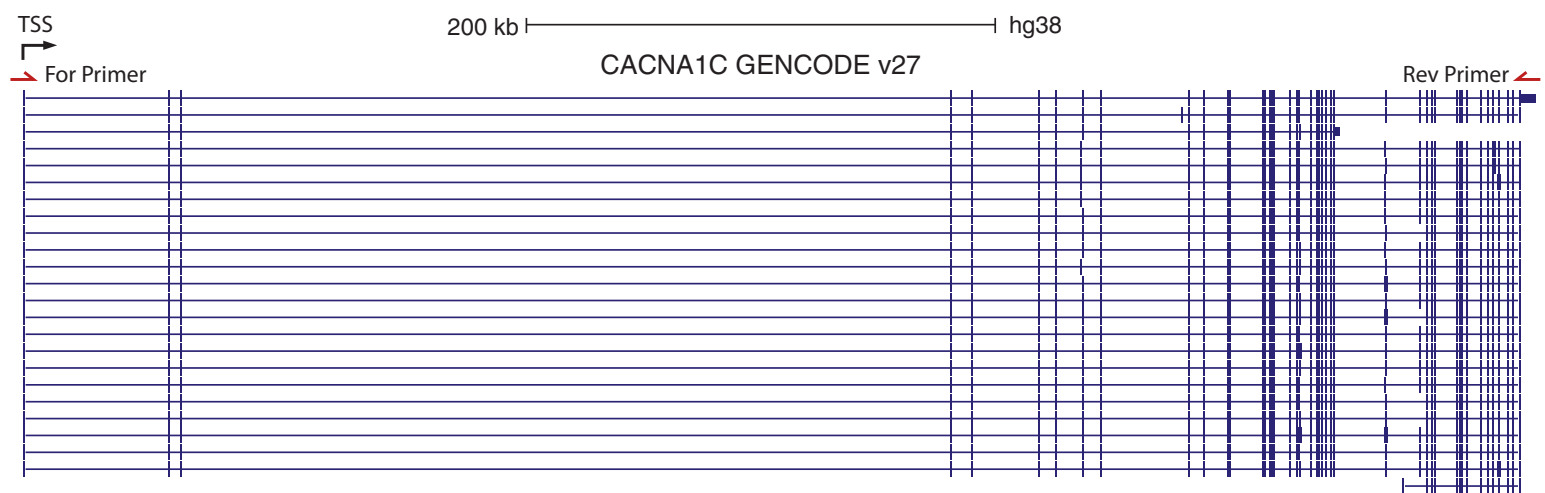

B Mean read quality per sequence length

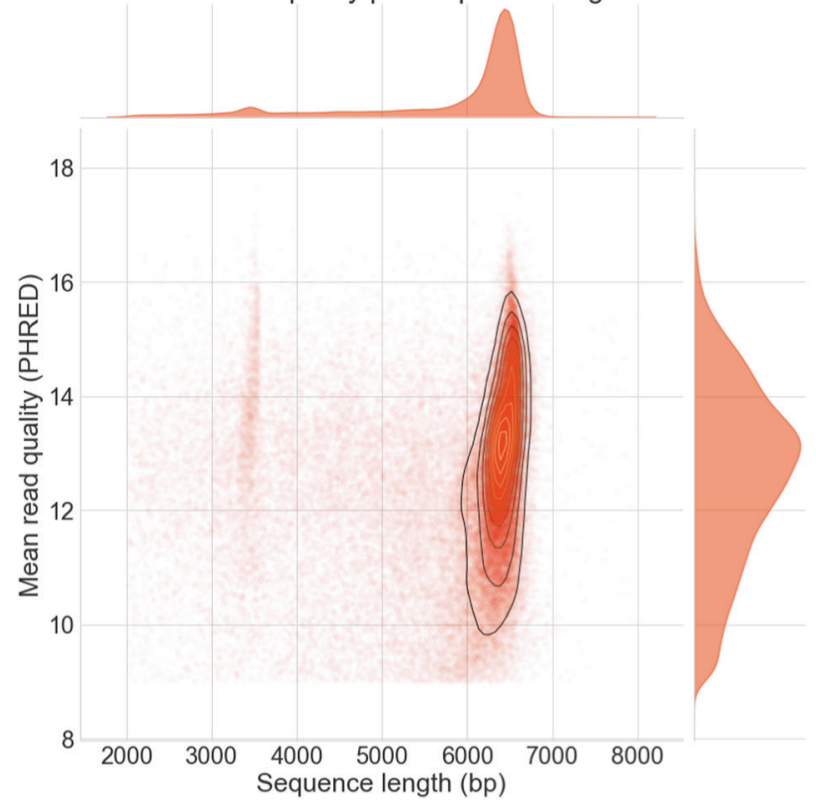

Fig. 1 Amplicon sequencing of CACNA1C. a UCSC genome browser screenshot of CACNA1C isoforms annotated in GENCODE V27. All transcripts in "Basic" annotated set shown. Black arrow shows direction of transcription. TSS transcription start site. Position of forward and reverse long-range PCR primers in first exon and

located within the expected intronic sequences, and at least six nucleotides away from existing exons. Novel exonic sequences were subsequently introduced to the CACNAIC metagene (concatenation of all exons) and reads were mapped again to this new model using GMAP to enable further characterization of alternative splicing and quantification of transcript expression.

To characterize novel exon junctions, we mapped all the reads to the human genome (hg38) using LAST v926 [39]. We identified all exon junctions that showed perfect and contiguous mapping (no sequencing errors nor insertion/deletions) of the read to the genome and used canonical acceptor and donor splice sites. We then used this comprehensive set of exon junctions to correct reads with sequencing errors at mapping
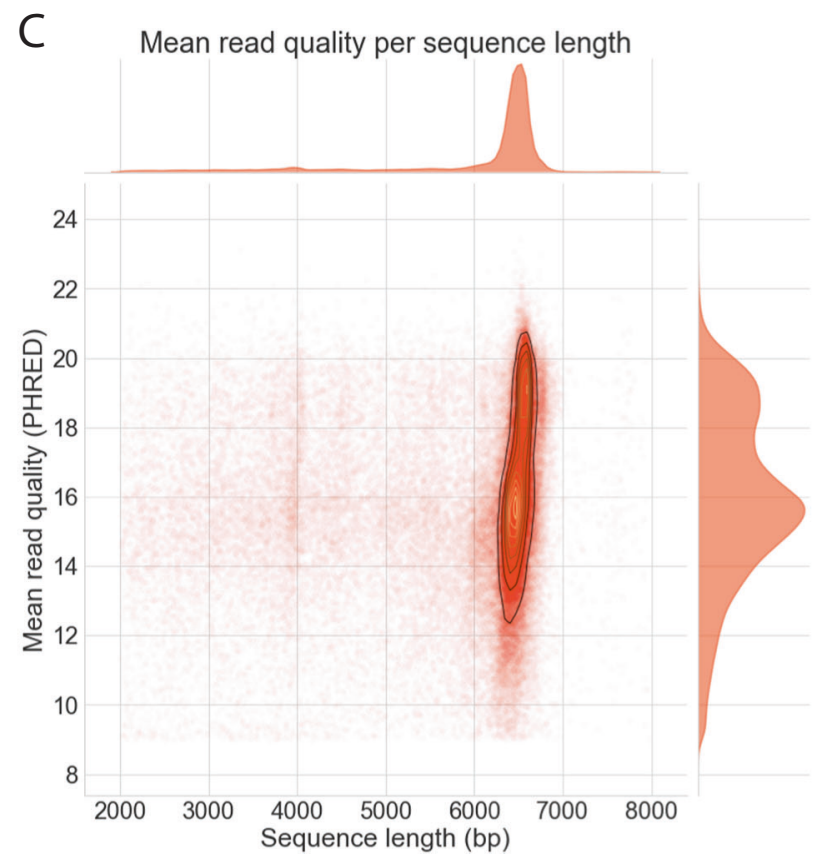

universal portion of final exon shown. Length vs Quality of all 2D pass reads from b Run $1 \mathbf{c}$ Run 2. Most reads are the full-length CACNA1C CDS. The presence of the $3.5 \mathrm{~kb}$ positive control CDS spike-in can be seen in Run1. Visualisation limited to reads between 2 and $8 \mathrm{~kb}$, encompassing $>98 \%$ of pass reads in each run

breakpoints by selecting the closest canonical splice site to the breakpoint.

To annotate transcripts that include novel exons and/or junctions, we parsed the CIGAR string from the alignment to the metagene, to identify the combination of exon junctions supported by each read. We subsequently clustered splicing patterns to annotate a unique set of isoforms for CACNAIC and enable their expression quantification. We applied a similar approach to annotate the transcripts containing novel splice sites. We filtered exons and transcripts of low abundance (i.e. supported only by one read), and clustered transcripts together to call only their longest possible variants.

Transcript expression both on exon and splice site levels was quantified using the number of reads supporting the 
transcript model. Reads mapping to multiple transcripts were down-weighted according to the number of transcripts they could be mapped to. Read counts were normalized across libraries using the trimmed Mean of M-values normalization method (TMM) [40]. For visualisation, all normalized counts were $\log _{10}$-transformed. Expression heatmaps and PCA plots were generated using R statistical language [41], by heatmap3 [42] and ggplot2 libraries [43], respectively. To normalise for the difference in sequencing depth between samples, we downsampled all libraries to match that with the smallest sequencing depth and recomputed transcript expression patterns.

\section{Quality control}

A subset of novel exons and novel exon junctions found in CACNAIC by nanopore sequencing (Supplementary Table 2) were confirmed by PCR targeting the novel sequence followed by Sanger sequencing (See Supplementary Information for details).

RNA extracted post-mortem shows variable degradation, and its quality is usually assessed using the RNA integrity number (RIN). In order to identify the minimum RIN required for reliable amplification of the full-length CDS of CACNAIC, RNA samples were artificially degraded (Supplementary Fig. 1). CACNAIC CDS amplification in these artificially degraded samples was assessed alongside that from striatal RNA samples of varying RINs from three adult donors (Supplementary Table 3).

\section{Results}

We successfully amplified and sequenced the full length $\sim 6.5 \mathrm{~kb}$ CACNAIC CDS (encompassing the full intron chain of $C A C N A 1 C$ ) from all samples (Fig. 1a). A minimum RIN of 6 is required, and a RIN of $>7$ is optimal, for amplification of full-length CACNAIC (Supplementary Fig. 1). Sequencing run 1 produced 112024 reads, including 52994 (47\%) 2D pass reads with an identified barcode. Run 2 produced 126314 reads, including 83221 (64\%) 2D pass reads with an identified barcode (Supplementary Tables 4 and 5). Most pass reads were full length, with the updated flowcell used in Run2 providing higher quality reads with a lower error rate (Fig. 1). All analyses were performed using the 2D pass barcoded reads, hereafter referred to simply as "reads".

\section{Long-range amplicon sequencing reveals many novel CACNA1C exons and isoforms}

We developed a bespoke alignment and mapping pipeline to maximise the transcript information obtained from nanopore sequencing reads, including the identification of novel exons, acceptor and donor splice sites, and splice junctions (Supplementary Fig. 2). Because of CACNAIC's complexity, we used two complementary approaches to identify transcripts: exon-level and splice-site-level analyses. These combined approaches identified a total of 251 unique CACNAIC transcript isoforms present in human brain, 241 of which are novel, including the use of novel exons, novel splice sites and junctions. The identity of these transcripts can be viewed on the UCSC Genome Browser: https://genome-euro.ucsc.edu/cgi-bin/hgTracks?hubUrl= https://opendata.earlham.ac.uk/opendata/data/CACNA1C_ isoforms $/$ hub.txt\&genome $=\mathrm{hg} 38$.

\section{Exon-level analysis}

We annotated a total of 39 potential novel exons within the CACNAIC locus, of which 38 were identified in at least 2 individuals or tissues and supported by at least 5 nanopore reads in each library (Fig. 2a; Supplementary Data Table 1).

We validated four out of four of the novel exons (selected across the range of abundance) by PCR and Sanger sequencing by confirming the splice junctions between the novel exon and its surrounding annotated exons. We also discovered a fifth novel exon that was spliced in between the targeted novel exon and the nearest known exon. This successful validation of a selection of novel exons provides high confidence that the novel exons identified by nanopore sequencing are real and actively incorporated into CACNAIC transcripts.

Integration of these 38 novel exons and the mapping of the reads to the metagene enabled the identification of novel and known (i.e. annotated) transcripts. Novel transcripts incorporate novel exons, and/or novel junctions between annotated exons and/or new combinations of known junctions. To limit the impact of sequencing errors on transcript annotations, we filtered the identified transcripts retaining only those found in at least 2 libraries with a minimum of 24 reads in total (see Supplementary Data Files 2 and 3 for transcript identity and abundance, respectively). We also created a high-confidence set of transcripts with an increased minimum threshold of 100 reads (see Supplementary Data Files 4 and 5 for transcript identity and abundance, respectively). Unless otherwise stated all analyses were performed on the high-confidence transcripts set. We identified 90 high-confidence CACNAIC transcripts across the 6 brain regions, including 7 previously annotated (GENCODE v27) and 83 novel (Supplementary Fig. 3). Seven of the novel high-confidence transcripts contained novel exons ( $1.8 \%$ of high-confidence reads in total), while the remaining 76 included previously undescribed junctions and junction combinations. 


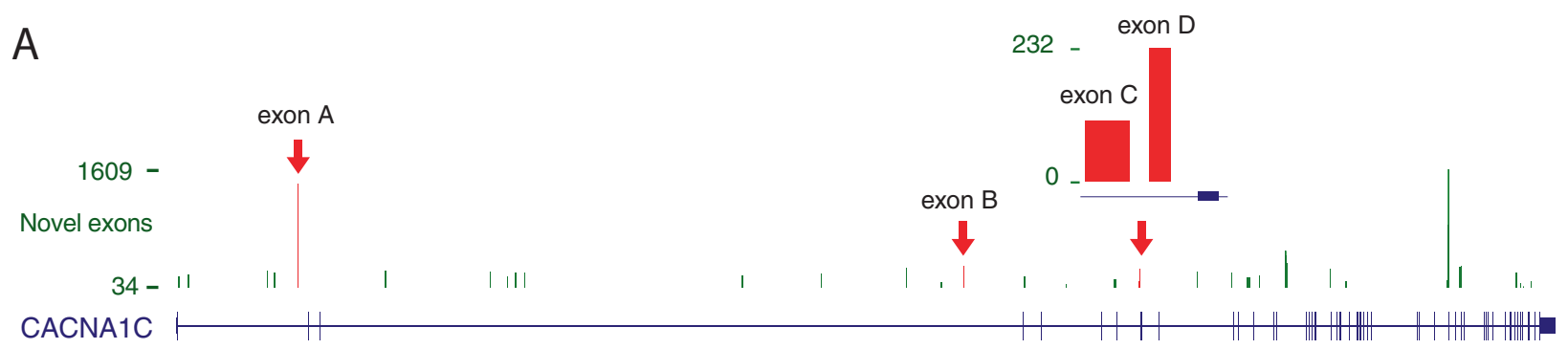

B

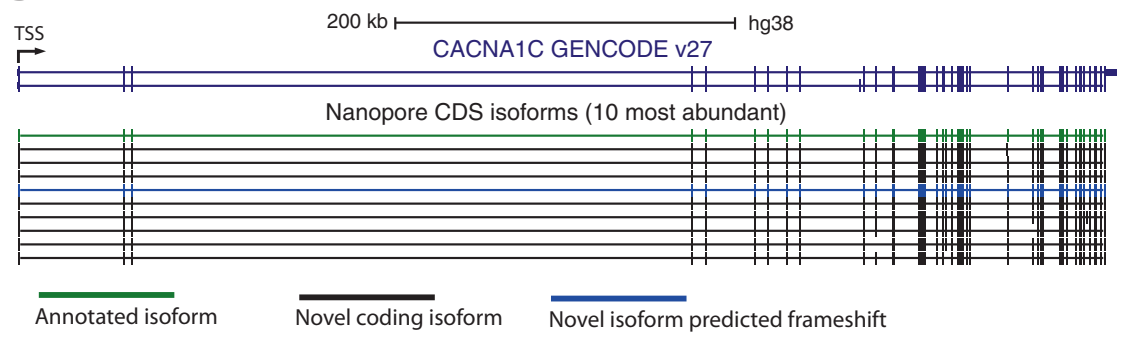

C

Fig. 2 a Annotation and read count for novel exonic sequences within CACNA1C. Red arrows indicate exons that have been validated (Supplementary Table 2). b, c Top 10 most abundant CACNA1C isoforms identified in brain using exon-level approach. b UCSC

As expected the most highly expressed transcript was previously annotated (ENST00000399641) and is supported by $40.2 \%(23.7-50 \%)$ of reads on average. In comparison, the most highly expressed novel transcript (CACNA1C $n 2199)$ represents on average $7.3 \%(2.7-25.3 \%)$ of all reads (Fig. 2b, c). Nine of the top ten expressed transcripts were novel, of which eight are predicted to maintain the $C A C$ $N A 1 C$ reading frame, suggesting a number of these most abundant novel transcripts encode functionally distinct protein isoforms (Fig. 2b, c). Without any thresholding, $75.5 \%$ of all the reads supported previously unannotated transcripts. These results suggest that novel CACNAIC transcripts are abundantly expressed as well as highly numerous, and that current annotations are missing many of the most abundant $C A C N A I C$ transcripts. Without filtering, we found evidence for the expression of only 18 of the current set of 31 annotated transcripts (GENCODE v27).

\section{Splice-site-level analysis}

The exon-level transcript identification approach described above provides a robust and conservative means to identify novel exons and to characterise full-length transcript structure. However, it is relatively insensitive to small-scale variation (of the order of a few amino acids). Therefore, we also implemented a splice junction-level analysis to annotate novel splice sites and junctions in the CACNAIC gene model. We used a conservative approach, which relies on the identification of junctions supported by error-free mapping at the junction, and the presence of canonical genome browser screenshot of top isoforms. Colours denote transcript type. c Proportion of high-confidence transcripts reads from the ten most abundant transcripts

splice sites. We thereby identified 497 novel splice sites, of which 393 were supported by at least 10 reads. Compared with previously annotated splice sites, novel donor and acceptor splice sites are less used (Supplementary Fig. 4). Including these splice sites, and after filtering for transcripts supported by at least 24 reads, we identified 195 transcripts, of which 111 are predicted to be coding (see Supplementary Data Files 6 and 7 for transcript identity and abundance, respectively). 28 of these were identical to transcripts identified by the exon-level analysis. Strikingly, most of the remainder (130) resulted from small-scale ( $\leq 15$ nucleotides) differences to our high-confidence exon-level transcripts, demonstrating the complementarity of these approaches. We validated the presence of specific small-scale deletions ( 3 and 4 amino acid microdeletions, described below) using Sanger sequencing.

\section{The expression profile of CACNA1C isoforms differs between brain regions}

We examined how CACNAIC isoform expression varies across brain regions and between individuals, focussing on transcripts identified using the exon-level approach (similar results were obtained using the splice-site-level transcripts [Supplementary Fig. 4]). We downsampled all libraries to 2729 (the smallest sequencing depth) prior to normalization. Differences between tissues is the main driver of the observed variation in expression between transcripts. Cerebellum and striatum were distinct from the four regions of the cortex (Fig. 3a, b), but expression was similar across 


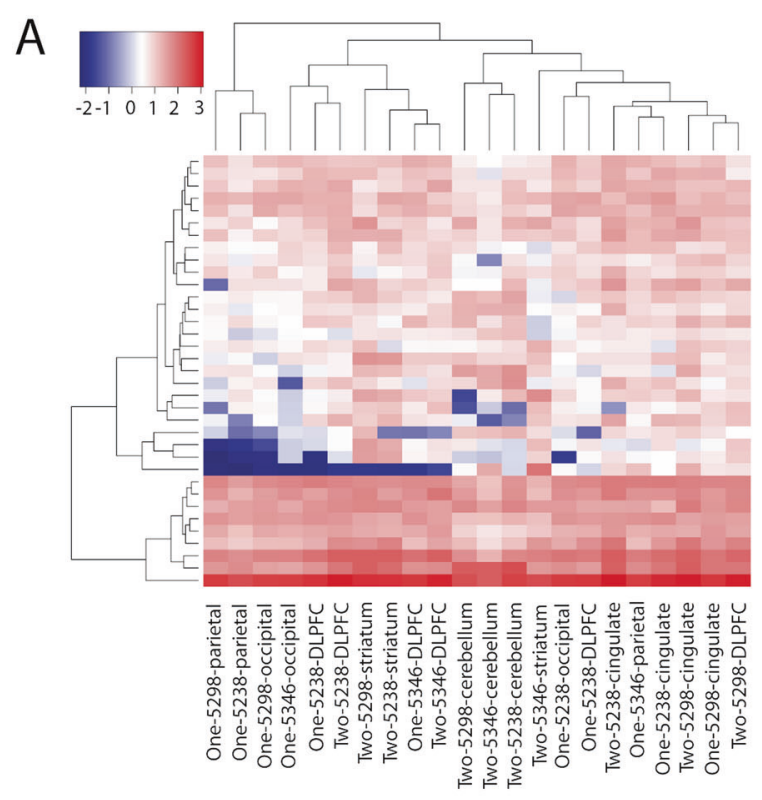

B
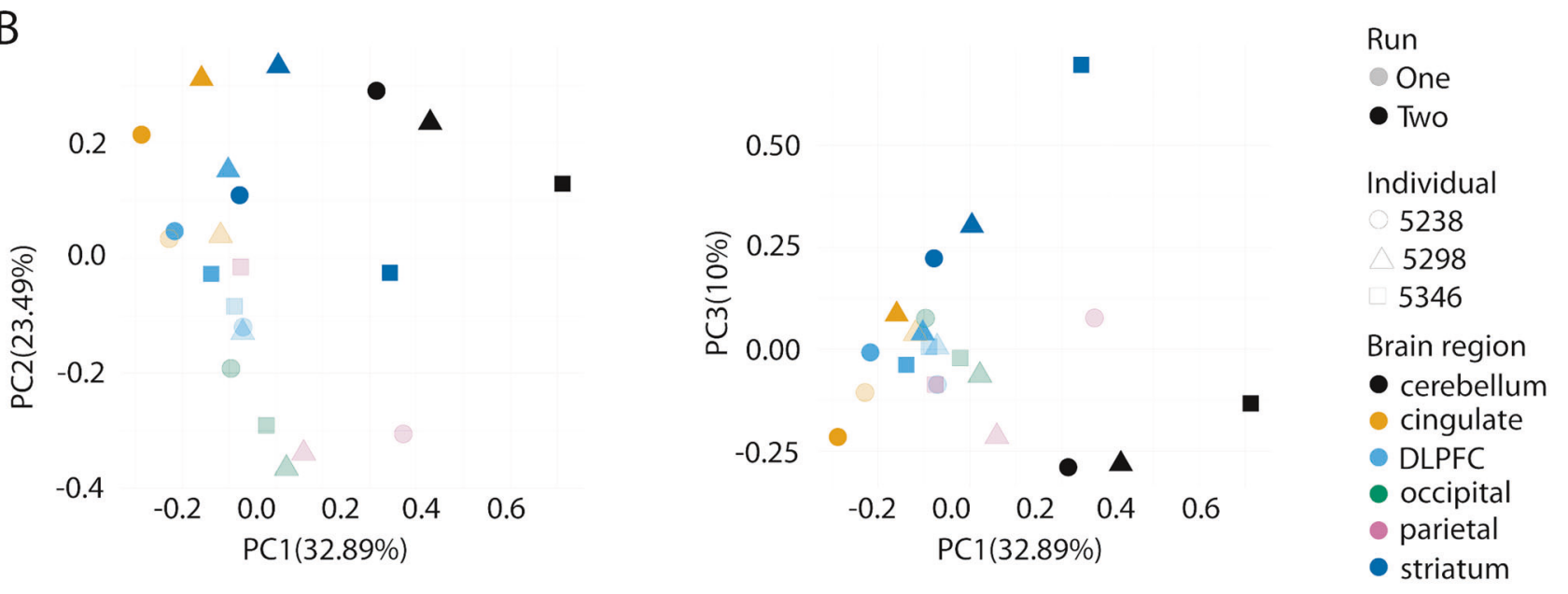

C
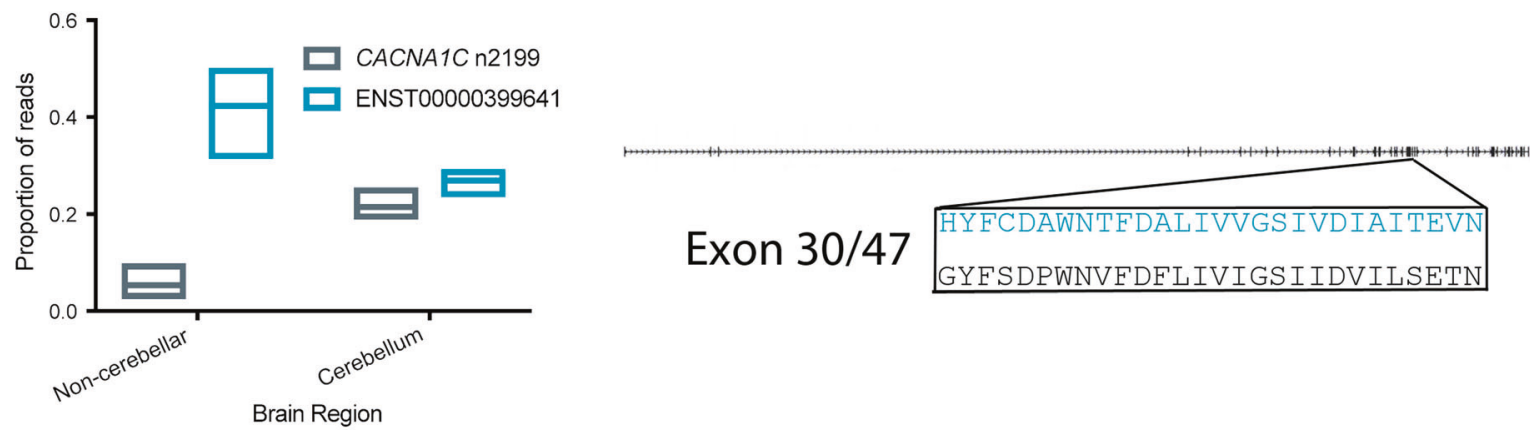

Fig. 3 Comparison of CACNA1C isoform expression between individuals and tissues. a Transcript expression levels (TPM) across tissues and individuals. "One" and "Two" denote sequencing runs. b Principal Component Analysis based on normalised transcript expression. c Isoform switching of ENST00000399641 and

individuals, consistent with observations at the transcriptome level [44]. The use of the more permissive filtered set of transcripts for expression estimation (see Methods)
CACNA1C n2199 in cerebellum. Left panel: box plots show minimum to maximum values with line at mean value. Right panel: the sequences of ENST00000399641 (blue) and CACNA1C n2199 (black) differ in the sequences of their 30th exons. The detail shows the amino acid sequences of this exon in the two transcripts

further improved the separation between regions, and highlighted potential differences in expression between cortical regions (Supplementary Figs. 5 and 6). 
Although we did not find any tissue-specific transcripts amongst our set of high-confidence transcripts, we observed a pronounced transcript expression switch in cerebellum. Outside of the cerebellum ENST00000399641 was the dominant transcript, whilst in cerebellum, ENST00000399641 and CACNA1C n2199 were expressed at similar levels (Fig. 3c).

\section{Predicted impact of novel isoforms on the $\mathrm{Ca}_{\mathrm{v}} \mathbf{1 . 2}$ protein model}

CACNAIC encodes the pore-forming $\mathrm{Ca}_{\mathrm{V}} 1.2$ alpha $_{1} \mathrm{VGCC}$ subunit. The calcium pore conmprises 24 transmembrane repeats, clustered into 4 domains linked by intracellular loops (Fig. 4a). Among the 83 novel exon-level transcripts we identified, 51 potentially encode functional $\mathrm{Ca}_{\mathrm{V}} 1.2$ channels, whilst the remainder are likely non-coding as they contain deletions in critical membrane-spanning regions and/or frameshifts. Notably, putatively coding isoforms represent $87.8 \%$ of total high-confidence reads, demonstrating that while non-coding transcripts are numerous, it is putatively coding transcripts that represent the vast majority of reads (Supplementary Fig. 7).

Around half of putatively coding exon-level transcripts (25 of 51; 26.8\% of total coding reads) consist of novel combinations of already annotated exons. The remainder include novel exons and/or deletions (Fig. 3b). Many of the novel splicing events are seen across multiple transcripts. For example, five transcripts $(2.8 \%$ on average, $5.2 \%$ maximally, of total coding reads) predict $\mathrm{Ca}_{\mathrm{V}} 1.2$ protein isoforms with an in-frame deletion in the intracellular I-II linker, and seven (6.9\% on average, $11.9 \%$ maximally, of total coding reads) contain an in-frame deletion in the IVS4IVS5 linker (Fig. 4a). Ten protein isoforms $(12.7 \%$, on average, $22.3 \%$, maximally, of total coding reads)including CACNA1C n1925, one of the three most abundant transcripts-include a previously described microdeletion in the extracellular IVS3-IVS4 linker (Fig. 4b) [33]. One also contains a 3 aa in-frame insertion in this same linker region, highlighting this region as a 'hotspot' for alternative

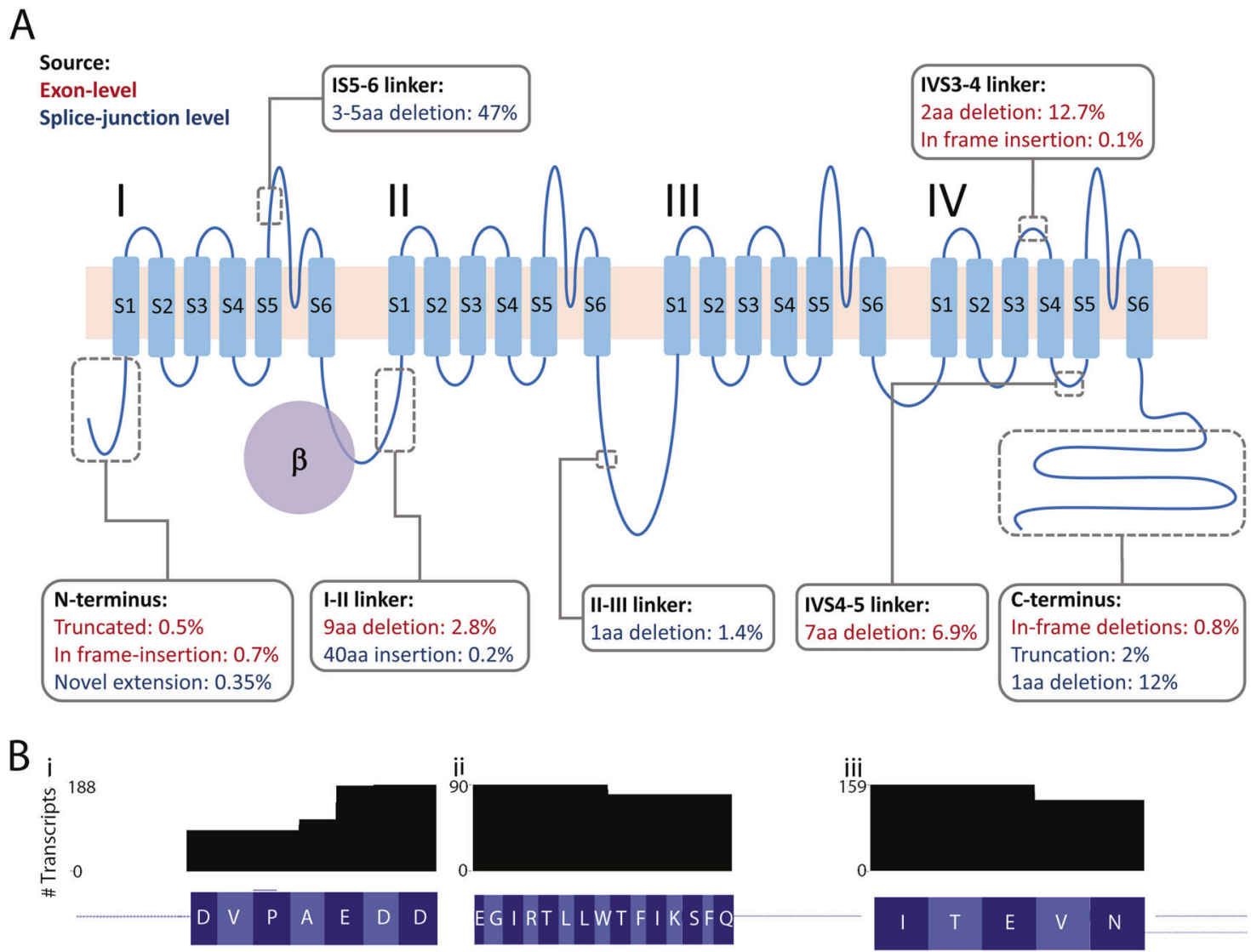

Fig. 4 Impact of novel splicing events on the CACNA1C protein model. a CACNA1C encodes the primary pore-forming subunit of the $\mathrm{Ca}_{\mathrm{V}}$ 1.2. $\mathrm{Ca}_{\mathrm{V}} 1.2$ is formed of four domains (I-IV), each comprised of six transmembrane domains (S1-S6), which are linked by intracellular loops. The obligate beta subunit binds to the I-II intracellular loop, as shown. Grey boxes indicate the location of novel, in-frame insertions and deletions, discussed in the main text. Values indicate the mean proportion of reads containing each variant. Where variants were identified using both analysis approaches, exon-level counts were used to derive abundance (red text); variants identified only using the splicesite-level approach are indicated with blue text. b Number of protein isoforms containing three microdeletions: (i) in the I-II linker, (ii) in the IV4-5 linker and (iii) the previously reported microdeletion in the IV3-4 linker 
splicing, of interest given the relevance of this linker for determining channel properties [33].

Several protein isoforms predict alternative $\mathrm{N}-$ and C-termini (Fig. 4a), which are involved in coupling $\mathrm{Ca}_{V} 1.2$ signalling to intracellular signalling cascades and calcium-dependent inactivation [31, 45]. Variation in these regions is predicted to affect a small fraction of the $\mathrm{Ca}_{\mathrm{v}} 1.2$ pool, but such protein isoforms may still be of biological significance if they show altered inactivation properties and/ or coupling to second messenger systems (Fig. 4a).

The splice-site-level analysis identified additional sites of variation (Fig. 4). Most strikingly, $47 \%$ of transcripts included an in-frame microdeletion of 3, 4 or 5 amino acids in the IS5-6 linker (corresponding to amino acids D306E310 in Uniprot entry Q13936). The 4 amino acid microdeletion was previously described in heart but not brain [29], but the 3 and 5 amino acid microdeletions are novel (Fig. 4b). Their functional impact is unknown but the S5-S6 linker is part of the pore-forming region and these microdeletions fall within a region previously implicated in determining channel conductance [46].

\section{Discussion}

We combined long-range RT-PCR with nanopore sequencing in order to characterize the full coding sequence from transcripts of CACNAIC. To our knowledge, we are the first to have used this approach successfully in human postmortem tissue. The vast majority of $C A C N A I C$ transcripts are novel, and many of these are abundant. We demonstrate marked differences in CACNAIC transcript profiles between brain regions, with the cerebellum in particular showing a notable switch in isoform abundance compared with cortex. Our results demonstrate that $C A C N A 1 C$ transcripts are much more diverse than previously appreciated, and emphasise the importance of studying full-length isoforms and of access to high-quality human brain tissue.

Despite annotations of very high quality and active curation [19, 47], an increasing number of studies report novel protein-coding sequences and exons in the human genome. The rapid development of long-read sequencing technologies opens the unique opportunity to gain an accurate representation of transcript diversity, as each read encompasses a full transcript. This knowledge is particularly critical for genes with complex models, for example a recent study of DSCAM, identified 18,496 transcripts out of over 19,000 of those theoretically possible [48]. We anticipate that long-read sequencing applications will further help decipher transcript expression and splicing, especially for genes expressed in the brain, which exhibits prominent use of alternative splicing and tissue-specific exons [14-18, 49].
Our study highlights the power of long-read sequencing for the annotation and characterisation of alternatively spliced transcripts. We demonstrate a five-fold increase in the number of annotated transcripts for $C A C N A 1 C$, identifying novel exons and deletions within the coding sequence, as well as novel combinations of previously annotated exons. Because of the high quality of the genomic assembly at the CACNAIC locus [50], the novel exons, and splice sites we describe are unlikely to result from mapping errors. Instead, our findings support those from transcriptome analyses that indicate that a significant proportion of gene isoforms in human brain remain unannotated [51]. Supporting their potential importance, a number of the novel transcripts are highly abundant individually, and collectively they encompass the majority of reads. Our finding that the vast majority of reads from novel transcripts maintain the CACNAIC reading frame also supports the hypothesis that these are functionally relevant and not simply products of "noisy" splicing. Furthermore, we identified several abundant in-frame deletions that are present in a number of transcripts; if translated these could dramatically impact on $\mathrm{Ca}_{\mathrm{V}} 1.2$ 's function in the cell $[29,30,52]$. Notably, a number of our novel predicted protein isoforms include alterations in domains known to be important for determining channel properties and coupling to second messenger systems $[29,31,53]$, thereby providing testable hypotheses as to their predicted functional impact. Now that the transcript structure of CACNAIC is clearer, it will also be of interest to examine the cumulative effect of functional variation across the $\mathrm{Ca}_{\mathrm{V}} 1.2$ protein on channel function. Conversely, we identified a relatively low number of annotated transcripts in our dataset. This is perhaps unsurprising, given that many of the currently annotated transcripts are likely predictions from ESTs and incomplete cDNAs. Taken together, these observations demonstrate the importance of long-read sequencing for the accurate characterisation of transcript structure and alternative splicing.

The need to understand the diversity of $\mathrm{Ca}_{\mathrm{V}} 1.2$ isoforms may be of clinical and therapeutic relevance. First, characterization of the complement of full-length CACNAIC isoforms is a necessary first step towards understanding how its transcript profile might be altered by a risk variant or disease state. Second, calcium channel blockers, which have $\mathrm{Ca}_{\mathrm{V}} 1.2$ as one of their primary targets, are licensed for cardiovascular indications and have possible utility as a therapeutic strategy for psychiatric disorders [54-57]. Since the $\mathrm{Ca}_{\mathrm{V}} 1.2$ proteins that arise from CACNAIC splicing show differential sensitivity to the existing calcium channel blockers [52] it may be possible to selectively target disease-relevant CACNAIC isoforms and/or those that are differentially expressed in the brain vs. the periphery, to 
provide novel psychotropic agents that are both more potent and are freer from peripheral side effects.

Our results indicate differences in the abundance, but not the identity, of CACNAIC transcripts between brain regions. Utilising our more conservative exon-level analysis, in most regions there is a single major CACNAIC isoform, with levels of expression almost five-fold higher than the second most highly expressed transcript. However, in the cerebellum there is a switch in transcript abundance, such that the two most abundant transcripts are expressed at similar levels to one another. The consistent nature of this switch in different individuals supports the hypothesis that this is a regulated switch in expression. In contrast, our analysis demonstrated only minor differences in the transcript profile between individuals (Fig. 3b). Given these findings, we are therefore confident that our analysis is likely to have identified the most common and abundant CACNAIC transcripts present in adult human brain. However, we anticipate that the relative abundance of these transcripts will likely differ between individuals. Our splice site-level analysis found broadly the same results, although there was no longer a single dominant isoform in each region, largely due to variation in the IS5-6 linker. While we have validated these variations, increased certainty in their abundance and impact on overall transcript structure will likely require further improvements in exon boundary identification with nanopore sequencing. The details of the CACNAIC transcript structure revealed here will facilitate the mining of existing large-scale RNA-Seq datasets to investigate these differences between individuals (and between subgroups based on e.g. genotype or disease status).

In summary, our findings demonstrate the utility of longrange amplicon sequencing for the identification and characterisation of gene isoform profiles. More specifically, they demonstrate that the human brain CACNAIC transcriptional profile is substantially more complex than currently appreciated. Our approach focused on annotated $5^{\prime}$ and $3^{\prime}$ ends; thus, there is a need for further in depth investigation, e.g. using RACE and capture sequencing. Understanding the functional consequences of this isoform diversity will advance our understanding of the role of VGCCs in the human brain and their involvement in psychiatric disorders [2-4]. Finally, some of the novel isoforms we present here may prove novel and selective therapeutic targets for these disorders [54].

\section{Data availability}

Nanopore sequencing reads generated and analysed in the present study are available from ENA (PRJEB34660). Transcript isoforms are available as supplementary data and can be direct visualised and downloaded via our UCSC Genome
Browser Track Hub (https://genome-euro.ucsc.edu/cgi-bin/ hgTracks?hubUrl=https://opendata.earlham.ac.uk/opendata/ data/CACNA1C_isoforms/hub.txt\&genome $=$ hg38).

Acknowledgements We are grateful to Li Chen and Arne Mould for technical assistance. This research was supported by a Wellcome Trust [201879/Z/16/Z] award to MBC and a UK Medical Research Council [MR/P026028/1] award to EMT. The authors wish to acknowledge the following funding sources: $\mathrm{MBC}$ is supported by an Australian National Health and Medical Research Council (NHMRC) Early Career Fellowship [APP1072662]. EMT is supported by a Royal Society University Research Fellowship. WH and TW are supported by the BBSRC, Institute Strategic Programme Grant [BB/J004669/1], BBSRC Core Strategic Programme Grant [BB/P016774/1]. This study was supported by the National Institute for Health Research Oxford Health Biomedical Research Centre. The human brain tissue repository is supported by the Lieber Institute for Brain Development. The views expressed are those of the authors and not necessarily those of the NHS, the NIHR or the Department of Health. The contents of the published material are solely the responsibility of the administering institution, a participating institution, or individual authors and do not reflect the views of NHMRC.

Author contributions MBC and EMT conceived the study. MBC, ABG, PJH, NAH, TW, WH and EMT designed experiments. MBC, $\mathrm{ABG}$ and EMT performed experiments and nanopore sequencing. ABG and NAH performed PCR validations. JEK, TH and DRW provided materials. TW and $\mathrm{WH}$ wrote the analysis pipeline and performed informatic analyses. $\mathrm{MBC}, \mathrm{NAH}, \mathrm{ABG}, \mathrm{TW}, \mathrm{WH}$ and EMT wrote the manuscript.

\section{Compliance with ethical standards}

Conflict of interest EMT and PJH are in receipt of an Unrestricted Educational Grant from J\&J Innovations to investigate $\mathrm{Ca}_{V} 1.2$ channels as therapeutic targets for psychiatric disorders. This grant did not fund the research detailed here. MBC, TW, NALH and WH have received support from Oxford Nanopore Technolgies (ONT) to present these and other findings at scientific conferences. However, ONT played no role in study design, execution, analysis or publication.

Publisher's note Springer Nature remains neutral with regard to jurisdictional claims in published maps and institutional affiliations.

Open Access This article is licensed under a Creative Commons Attribution 4.0 International License, which permits use, sharing, adaptation, distribution and reproduction in any medium or format, as long as you give appropriate credit to the original author(s) and the source, provide a link to the Creative Commons license, and indicate if changes were made. The images or other third party material in this article are included in the article's Creative Commons license, unless indicated otherwise in a credit line to the material. If material is not included in the article's Creative Commons license and your intended use is not permitted by statutory regulation or exceeds the permitted use, you will need to obtain permission directly from the copyright holder. To view a copy of this license, visit http://creativecommons. org/licenses/by/4.0/.

\section{References}

1. Corvin A, Sullivan PF. What next in schizophrenia genetics for the psychiatric genomics consortium? Schizophrenia Bull. 2016; 42:538-41. 
2. Schizophrenia Working Group of the Psychiatric Genomics Consortium. Biological insights from 108 schizophreniaassociated genetic loci. Nature. 2014;511:421-7.

3. Cross-Disorder Group of the Psychiatric Genomics Consortium. Identification of risk loci with shared effects on five major psychiatric disorders: a genome-wide analysis. Lancet Lond Engl. 2013;381:1371-9.

4. Stahl EA, Breen G, Forstner AJ, McQuillin A, Ripke S, Trubetskoy V et al. Genome-wide association study identifies 30 loci associated with bipolar disorder. Nat Genet. 2019;51:793-803.

5. Furlanis E, Traunmüller L, Fucile G, Scheiffele P. Landscape of ribosome-engaged transcript isoforms reveals extensive neuronalcell-class-specific alternative splicing programs. Nat Neurosci. 2019;22:1709-17.

6. Li YI, van de Geijn B, Raj A, Knowles DA, Petti AA, Golan D, et al. RNA splicing is a primary link between genetic variation and disease. Science. 2016;352:600-4.

7. Jaffe AE, Straub RE, Shin JH, Tao R, Gao Y, Collado-Torres L, et al. Developmental and genetic regulation of the human cortex transcriptome illuminate schizophrenia pathogenesis. Nat Neurosci. 2018;21:1117-25.

8. Li M, Jaffe AE, Straub RE, Tao R, Shin JH, Wang Y, et al. A human-specific AS3MT isoform and BORCS7 are molecular risk factors in the 10q24.32 schizophrenia-associated locus. Nat Med. 2016;22:649-56.

9. Hughes T, Hansson L, Sønderby IE, Athanasiu L, Zuber V, Tesli $\mathrm{M}$, et al. A loss-of-function variant in a minor isoform of ANK3 protects against bipolar disorder and schizophrenia. Biol Psychiatry. 2016;80:323-30.

10. Tao R, Cousijn H, Jaffe AE, Burnet PWJ, Edwards F, Eastwood SL, et al. Expression of ZNF804A in human brain and alterations in schizophrenia, bipolar disorder, and major depressive disorder: a novel transcript fetally regulated by the psychosis risk variant rs1344706ZNF804A expression in human brainZNF804A expression in human brain. JAMA Psychiatry. 2014;71:1112-20.

11. Paterson C, Wang Y, Hyde WM, Weinberger DR, Kleinman JE, Law AJ. Temporal, diagnostic, and tissue-specific regulation of NRG3 isoform expression in human brain development and affective disorders. Am J Psychiatry. 2017;174:256-65.

12. Oldmeadow C, Mossman D, Evans T-J, Holliday EG, Tooney PA, Cairns MJ, et al. Combined analysis of exon splicing and genome wide polymorphism data predict schizophrenia risk loci. J Psychiatr Res. 2014;52:44-9.

13. Gandal MJ, Zhang P, Hadjimichael E, Walker RL, Chen C, Liu S, et al. Transcriptome-wide isoform-level dysregulation in ASD, schizophrenia, and bipolar disorder. Science. 2018;362:eaat8127.

14. Tapial J, Ha KCH, Sterne-Weiler T, Gohr A, Braunschweig U, Hermoso-Pulido A, et al. An atlas of alternative splicing profiles and functional associations reveals new regulatory programs and genes that simultaneously express multiple major isoforms. Genome Res. 2017;27:1759-68.

15. Mele M, Ferreira PG, Reverter F, DeLuca DS, Monlong J, Sammeth $\mathrm{M}$, et al. Human genomics. The human transcriptome across tissues and individuals. Science. 2015;348:660-5.

16. Jensen KB, Dredge BK, Stefani G, Zhong R, Buckanovich RJ, Okano HJ, et al. Nova-1 regulates neuron-specific alternative splicing and is essential for neuronal viability. Neuron. 2000;25:359-71.

17. Yang YY, Yin GL, Darnell RB. The neuronal RNA-binding protein Nova-2 is implicated as the autoantigen targeted in POMA patients with dementia. Proc Natl Acad Sci USA. 1998;95:13254-9.

18. Raj B, Blencowe BJ. Alternative splicing in the mammalian nervous system: recent insights into mechanisms and functional roles. Neuron. 2015;87:14-27.

19. Harrow J, Frankish A, Gonzalez JM, Tapanari E, Diekhans M, Kokocinski F, et al. GENCODE: the reference human genome annotation for The ENCODE Project. Genome Res. 2012; 22:1760-74.

20. Li YI, Sanchez-Pulido L, Haerty W, Ponting CP. RBFOX and PTBP1 proteins regulate the alternative splicing of micro-exons in human brain transcripts. Genome Res. 2015;25:1-13.

21. Irimia M, Weatheritt RJ, Ellis JD, Parikshak NN, GonatopoulosPournatzis $\mathrm{T}$, Babor $\mathrm{M}$, et al. A highly conserved program of neuronal microexons is misregulated in autistic brains. Cell. 2014;159:1511-23.

22. Steijger T, Abril JF, Engstrom PG, Kokocinski F, Consortium R, Hubbard TJ, et al. Assessment of transcript reconstruction methods for RNA-seq. Nat Methods. 2013;10:1177-84.

23. Hardwick SA, Chen WY, Wong T, Deveson IW, Blackburn J, Andersen SB, et al. Spliced synthetic genes as internal controls in RNA sequencing experiments. Nat Methods. 2016;13:792-8.

24. Green EK, Hamshere M, Forty L, Gordon-Smith K, Fraser C, Russell E, et al. Replication of bipolar disorder susceptibility alleles and identification of two novel genome-wide significant associations in a new bipolar disorder case-control sample. Mol Psychiatr. 2013;18:1302-7.

25. Psychiatric Genomics Consortium Bipolar Working Group. Largescale genome-wide association analysis of bipolar disorder identifies a new susceptibility locus near ODZ4. Nat Genet. 2011;43:977-83.

26. Heyes S, Pratt WS, Rees E, Dahimene S, Ferron L, Owen MJ, et al. Genetic disruption of voltage-gated calcium channels in psychiatric and neurological disorders. Prog Neurobiol. 2015;134:36-54.

27. Yang Y, Chen X, Margulies K, Jeevanandam V, Pollack P, Bailey $\mathrm{BA}$, et al. L-type $\mathrm{Ca} 2+$ channel $\alpha 1 \mathrm{cSubunit}$ isoform switching in failing human ventricular myocardium. J Mol Cell Cardiol. 2000;32:973-84.

28. Wang D, Papp AC, Binkley PF, Johnson JA, Sadee W. Highly variable mRNA expression and splicing of L-type voltagedependent calcium channel alpha subunit $1 \mathrm{C}$ in human heart tissues. Pharmacogenetics genomics. 2006;16:735-45.

29. Tang ZZ, Liang MC, Lu S, Yu D, Yu CY, Yue DT, et al. Transcript scanning reveals novel and extensive splice variations in human 1type voltage-gated calcium channel, Cav1.2 alphal subunit. J Biol Chem. 2004;279:44335-43.

30. Hofmann F, Flockerzi V, Kahl S, Wegener JW. L-type CaV1.2 calcium channels: from in vitro findings to in vivo function. Physiol Rev. 2014;94:303-26.

31. Striessnig J, Pinggera A, Kaur G, Bock G, Tuluc P. L-type Ca(2 + ) channels in heart and brain. Wiley Interdiscip Rev Membr Transp Signal. 2014;3:15-38.

32. Bolisetty MT, Rajadinakaran G, Graveley BR. Determining exon connectivity in complex mRNAs by nanopore sequencing. Genome Biol. 2015;16:204.

33. Weirather JL, de Cesare M, Wang Y, Piazza P, Sebastiano V, Wang XJ, et al. Comprehensive comparison of Pacific Biosciences and Oxford Nanopore Technologies and their applications to transcriptome analysis. F1000Res. 2017;6:100.

34. Byrne A, Beaudin AE, Olsen HE, Jain M, Cole C, Palmer T, et al. Nanopore long-read RNAseq reveals widespread transcriptional variation among the surface receptors of individual B cells. Nat Commun. 2017;8:16027.

35. Colantuoni C, Lipska BK, Ye T, Hyde TM, Tao R, Leek JT, et al. Temporal dynamics and genetic control of transcription in the human prefrontal cortex. Nature. 2011;478:519.

36. Melé M, Ferreira PG, Reverter F, DeLuca DS, Monlong J, Sammeth $\mathrm{M}$, et al. The human transcriptome across tissues and individuals. Science. 2015;348:660-5.

37. Wang M, Kong L. pblat: a multithread blat algorithm speeding up aligning sequences to genomes. BMC Bioinforma. 2019;20:28.

38. Wu TD, Watanabe CK. GMAP: a genomic mapping and alignment program for mRNA and EST sequences. Bioinformatics. 2005;21:1859-75. 
39. Kielbasa SM, Wan R, Sato K, Horton P, Frith MC. Adaptive seeds tame genomic sequence comparison. Genome Res. 2011;21:487-93.

40. Robinson MD, Oshlack A. A scaling normalization method for differential expression analysis of RNA-seq data. Genome Biol. 2010;11:R25.

41. R Development Core Team. R: a language and environment for statistical computing. Vienna, Austria: R Development Core Team; 2008.

42. Zhao S, Guo Y, Sheng Q, Shyr Y. Heatmap3: an improved heatmap package with more powerful and convenient features. BMC Bioinforma. 2014;15:P16.

43. Wickham H. ggplot2: elegant graphics for data analysis. New York: Springer-Verlag; 2009.

44. GTex Consortium. Human genomics. The genotype-tissue expression (GTEx) pilot analysis: multitissue gene regulation in humans. Science. 2015;348:648-60.

45. Christel C, Lee A. Ca2+-dependent modulation of voltage-gated Ca2+ channels. Biochim Biophys Acta. 2012;1820:1243-52.

46. Dirksen RT, Nakai J, Gonzalez A, Imoto K, Beam KG. The S5-S6 linker of repeat I is a critical determinant of L-type $\mathrm{Ca} 2+$ channel conductance. Biophys J. 1997;73:1402-9.

47. Wright JC, Mudge J, Weisser H, Barzine MP, Gonzalez JM, Brazma A, et al. Improving GENCODE reference gene annotation using a high-stringency proteogenomics workflow. Nat Commun. 2016;7:11778.

48. Sun W, You X, Gogol-Doring A, He H, Kise Y, Sohn M, et al. Ultra-deep profiling of alternatively spliced Drosophila Dscam isoforms by circularization-assisted multi-segment sequencing. EMBO J. 2013;32:2029-38.

49. Gupta I, Collier PG, Haase B, Mahfouz A, Joglekar A, Floyd T, et al. Single-cell isoform RNA sequencing characterizes isoforms in thousands of cerebellar cells. Nat Biotechnol. 2018;36:1197.

50. Ebbert MTW, Jensen TD, Jansen-West K, Sens JP, Reddy JS, Ridge PG, et al. Systematic analysis of dark and camouflaged genes reveals disease-relevant genes hiding in plain sight. Genome Biol. 2019;20:97.

51. Tilgner H, Jahanbani F, Blauwkamp T, Moshrefi A, Jaeger E, Chen $\mathrm{F}$, et al. Comprehensive transcriptome analysis using synthetic long-read sequencing reveals molecular co-association of distant splicing events. Nat Biotechnol. 2015;33:736-42.

52. Welling A, Ludwig A, Zimmer S, Klugbauer N, Flockerzi V, Hofmann F. Alternatively spliced IS6 segments of the alpha 1C gene determine the tissue-specific dihydropyridine sensitivity of cardiac and vascular smooth muscle L-type $\mathrm{Ca} 2+$ channels. Circ Res. 1997;81:526-32.

53. Kaur G, Pinggera A, Ortner NJ, Lieb A, Sinnegger-Brauns MJ, Yarov-Yarovoy $\mathrm{V}$, et al. A polybasic plasma membrane binding motif in the I-II linker stabilizes voltage-gated CaV1.2 calcium channel function. J Biol Chem. 2015;290:21086-21100.

54. Cipriani A, Saunders K, Attenburrow MJ, Stefaniak J, Panchal P, Stockton S, et al. A systematic review of calcium channel antagonists in bipolar disorder and some considerations for their future development. Mol Psychiatry. 2016;21:1324-32.

55. Hayes JF, Lundin A, Wicks S, Lewis G, Wong ICK, Osborn DPJ, et al. Association of hydroxylmethyl glutaryl coenzyme A reductase inhibitors, L-type calcium channel antagonists, and biguanides with rates of psychiatric hospitalization and self-harm in individuals with serious mental illnessrepurposing HMG-CoA reductase inhibitors, L-type calcium channel antagonists, and biguanides for serious mental illnessrepurposing hmg-coa reductase inhibitors, L-type calcium channel antagonists, and biguanides for serious mental illness. JAMA Psychiatry. 2019;76:382-90.

56. Dubovsky SL. Applications of calcium channel blockers in psychiatry: pharmacokinetic and pharmacodynamic aspects of treatment of bipolar disorder. Expert Opin Drug Metab Toxicol. 2019;15:35-47.

57. Harrison PJ, Tunbridge EM, Dolphin AC, Hall J Voltage-gated calcium channel blockers for psychiatric disorders: genomic reappraisal. Br J Psychiatry 2019;24:1-4. 